\title{
RATIONALE OF PROSTHETICALLY-DRIVEN IMPLANT PLACEMENT UTILIZING IMPLANT- NAVIGATION SYSTEM: ACCURACY VALIDATION TRIAL
}

\author{
Amr H. Elkhadem* and Mostafa Helmy Mostafa Ahmed*
}

\begin{abstract}
Use of virtual reality simulation (VRS) settled a new era in dentistry, where a pre-operative virtual planning along with a surgical guide might benefit obtaining a prosthetically-driven precise implant positioning, particularly in problematic clinical scenarios.

Aim: A postulation aiming accuracy validation of the navigation system versus static guide technique for proper implant positioning.

Materials \& methods: Regarding this clinical study, 14 patients were recruited with bounded partially edentulous spans in the maxilla. The patients were randomly assorted into 2 groups. The first group (control) is the static guide group (SG), while the second group (test group) is the dynamic navigation group (DN). After implant installation, a postoperative CBCT was obtained $\&$ the obtained image was superimposed over the original implant plan to reveal any deviation between previously proposed plan and actual implant position.
\end{abstract}

Results: Regarding linear deviation between previously proposed plan and actual implant position in both groups measured at both the coronal \& apical areas, independent sample $t$ test revealed statistically insignificant difference among the comparative groups. On the other hand, regarding the angular deviation, independent sample $t$ test presented a statistically significant difference among the comparative groups.

Conclusion: The navigating Implantology system affords extremely precise navigation with diminished noticeable error regarding implant positioning. Furthermore, it permits properly transferred planning in an accurate prosthetically-driven manner.

KEYWORDS: Navigation, Computer-guided, Implant placement, Accuracy.

\section{INTRODUCTION}

Dental implants turn out to be a conventional mean in restoring missing teeth. However, implant positioning must be congruent with the proposed prosthetic rehabilitation. If not, aimed successful outcomes might not be achieved. ${ }^{[1,2]}$

\footnotetext{
* Associate Professor, Faculty of Dentistry, Cairo University.
} 
Dental implantology proposed new materials and innovative technologies, which gathers traditional approaches and new teaching plans, for example the virtual reality systems. ${ }^{[3-5]}$

Different approaches have been created allowing for better assessment of the bone proportions, utilizing computer tomography (CT) or digital volume tomography (DVT). ${ }^{[6-12]}$

While dental implants proven to be an affordable treatment modality, their conductivity in problematic cases with bone insufficiency became more efficient. In addition, to achieve accurate and secured implant poisoning, computer-aided techniques have been established. ${ }^{[13-15]}$

Progresses of virtual reality simulation (VRS) approaches enable working in a more genuine environment that nearly approximates the actual practice with more predictable results. ${ }^{[16-18]}$ To clarify, dental implantation assisted by navigation were established to enhance the precision less dangerous implant poisoning. ${ }^{[19-24]}$

Navigation could be performed in partially and completely edentulous cases for proper and secured implant poisoning. This represents an added value to overcome any unnecessary functional loading over the implants. Moreover, providing less possibility of dangerous encroachment onto any of adjacent anatomic structures. ${ }^{[25-29]}$

Several authors declared that, computerized navigation approaches enhance safety measurements in most of implant positioning modalities. ${ }^{[30-34]}$

Null hypothesis was postulated that no significant outcomes will be obtained when utilizing VRS regarding implant placement accuracy, throughout the entire examination period.

\section{MATERIALS AND METHODS}

In this clinical study, fourteen patients were recruited with bounded partially edentulous spans in the maxilla. The patients were randomly assorted into 2 groups. The first group (control) is the static guide group (SG), while the second group (test group) is the dynamic navigation group (DN). The patients required replacement of the missing teeth with an implant supported restoration.

For the control group (SG), every patient was scanned using CBCT scanning machine ${ }^{*}$ and the patient model was scanned using an optical scanner ${ }^{* *}$ $\&$ The virtual plan was made using the BlueSky plan software $^{* * *}$. The three-dimensional implant position was determined based on virtual tooth setup to obtain the best possible prosthetic driven implant location. Additionally, the dimensions of the implants used were adjusted based on the available bone volume and the proximity of vital landmarks.

After finalizing the plan, a 3D implant guide was designed with guide sleeve holes to guide the implant drilling and installation. The guide was printed using an LCD printer ${ }^{* * * *}$. The printed guide was cleaned with isopropyl alcohol, cured and the metal sleeves were fixed with resin cement. (Fig. 1-3)

The guide was checked for seating and fit inside the patient mouth.The drilling sequence of the guided $\mathrm{kit}^{* * * * *}$ was followed according to the manufacturer instruction. The implants were inserted through the guide till the final seating position.

In the dynamic navigation group (DN), the DENTCAM tracking system ${ }^{\#}$ was utilized. The system is composed of a tracking camera mounted on the surgical handpiece, a calibration device for the drills, and a tracking computer with a monitor

\footnotetext{
* Planmeca, Finland.

** Dental wings scanner 7, Dental wings Inc, Canada.

*** BlueSky Bio, 800 Liberty Drive. Libertyville, IL 60048, USA.

**** Phrozen shuffle XL, Taiwan.

***** Simple guide plus, Dentis Co, Korea.

\# DENACAM ${ }^{\circledR}$ System, mininavident, Gerberstrasse 5, 4410 Liestal, Switzerland.
} 


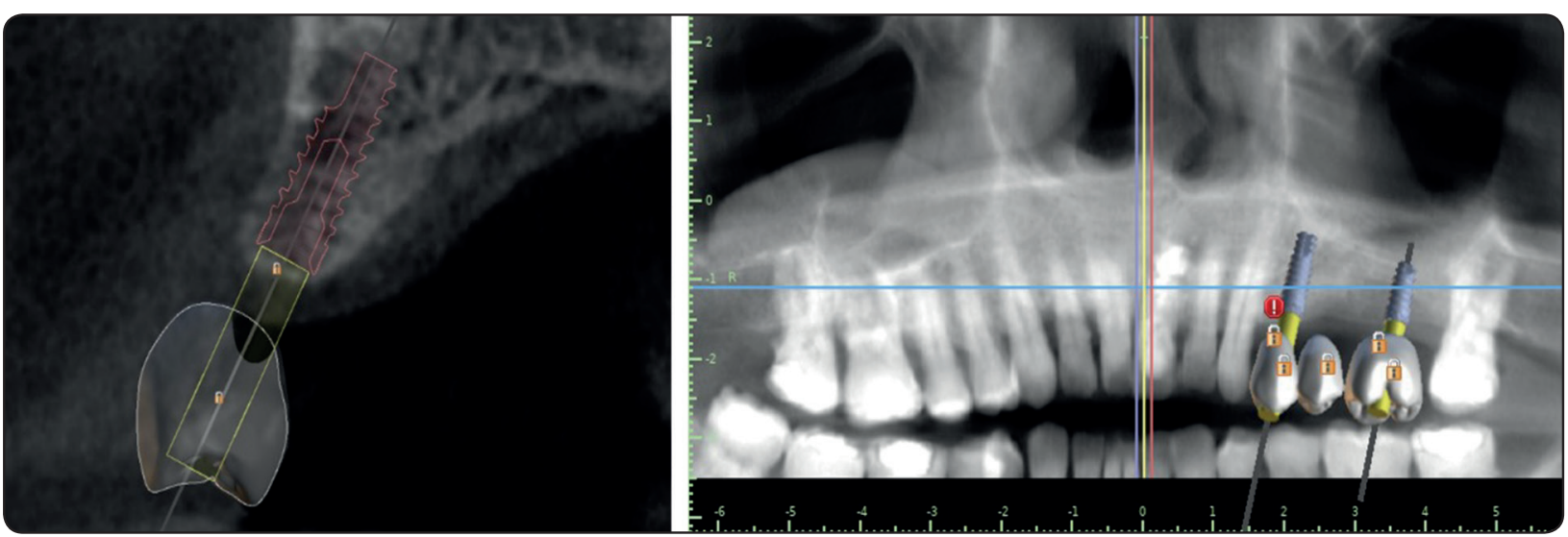

Fig. (1): The virtual plan using the BlueSky plan software.

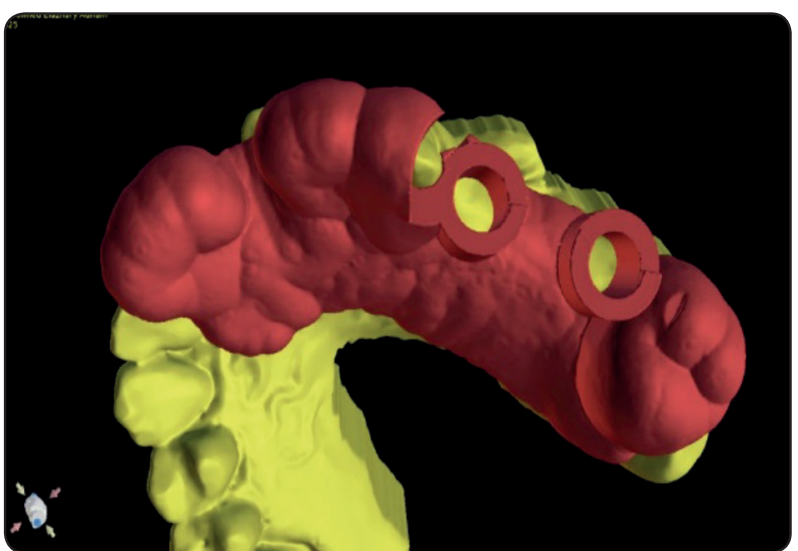

Fig. (2): The 3D implant guide with guide sleeve holes.

mounted over the dental unit. The computer contains the Dentcam tracking software.

The workflow for using the Dentcam navigation starts with a CBCT scan for the patient while wearing a small sectional tray with DENTMARK marker. The marker is basically a rectangular structure with laser engraved optical pattern composed of rectangles and circles brought to a ceramic substrate of $10 \times 15 \mathrm{~mm}$ in size. Two holes in the ceramic substrate guarantee an obvious nondisturbed identification of the position of the marker within the $\mathrm{CBCT}$ images.

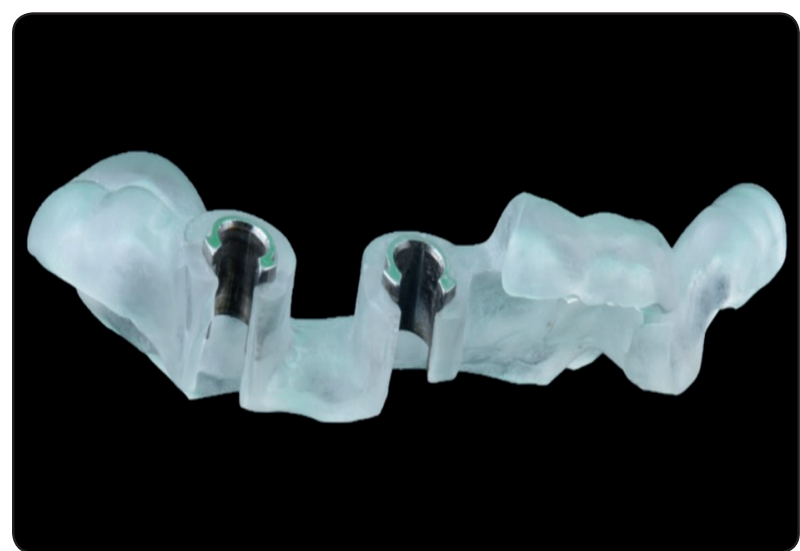

Fig. (3): The Final printed guide.

The tray with the marker was customized and secured to the patient teeth using putty and light wash addition silicone*.

The DICOM of the obtained CBCT was manipulated using the Osseoview** implant planning software. The three-dimensional implant position was determined based on virtual tooth setup to obtain the best possible prosthetically-driven implant location. Furthermore, the dimensions of the implants used were adjusted based on the available bone volume and the proximity of vital landmarks. The final 3D plan was saved and imported to the Dentcam tracking software. (Fig.4)

\footnotetext{
* Panasil, Katzenbach, Germany.

** 3D Diagnostix, USA.
} 
At the surgical stage, the marker used for the CBCT was replaced over the patient teeth depending on the fit of the used silicone index. The tracking camera was mounted over the handpiece, so that the camera can be rotated along the handpiece long axis.

The best location that allows direct un-obstructed view of the tracking marker was adjusted and then the camera location was then locked. This location varied according to the implant surgical site as well as the operator grip. Once the camera position has been locked, the initial drill was mounted on the handpiece and the tip of the drill was then placed to touch the base of the calibration tool.

The tracking software was able to identify the bur dimensions and calculate the zero point for the drill entry. The drill is then translated to the surgical

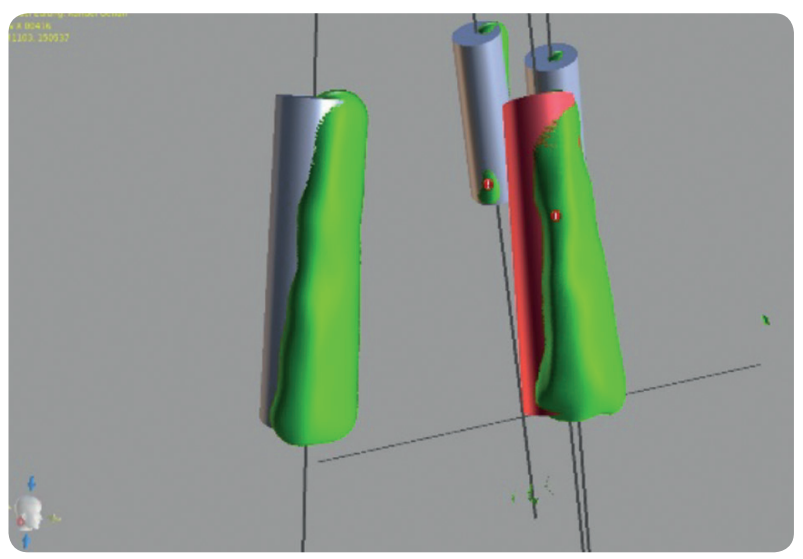

Fig. (4): The final 3D implant plan. site close to the proposed position. The software started to give live streaming of the drill movements relative to the original plan. (Fig.5)

Once the drill tip reached the proposed point of entry, the software activated a green circle and indicated the amount of linear and angular deviation from the plan.

The operator began to rotate the handpiece to obtain the best possible direction and then started the drilling. The drilling proceeded the software indicated the drilling depth till the final depth was reached. The same protocol of drill calibration and image guided drilling was continued for the whole drilling sequence. Finally, the implant was mounted on a rotary implant driver, calibrated, and inserted in the same navigation pattern. (Fig. 6)

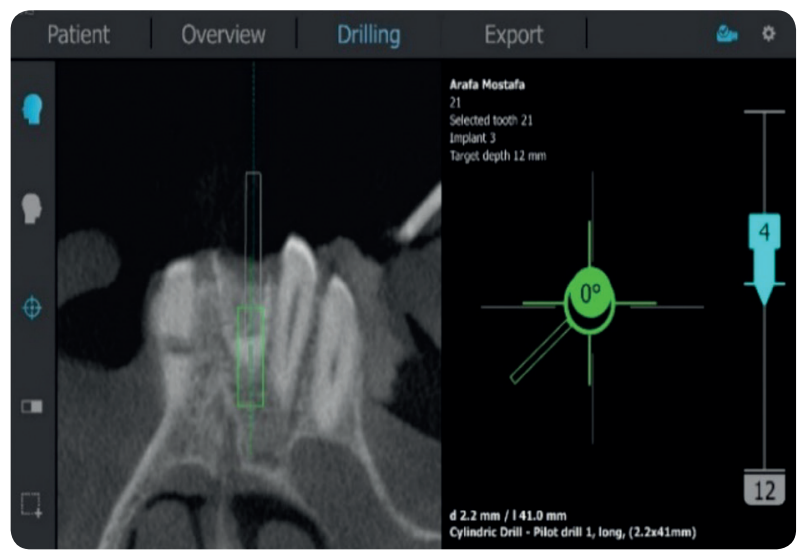

Fig. (5): Drilling appearance on the navigation system.



Fig. (6): Drilling system Intraorally. 



Fig. (7): Superimposition of the CBCT images over the original implant plan.

After implant installation, a postoperative CBCT was obtained \& the obtained image was superimposed over the original implant plan to establish both linear and angular deviancy between previously proposed plan and actual implant position. (Fig.7)

\section{RESULTS}

The linear variation between previously proposed plan and exact implant position in both groups was measured at both the coronal apical areas. For the coronal deviation the SG group showed a mean of $0.63 \pm 0.26 \mathrm{~mm}$ compared to $0.72 \pm 0.28 \mathrm{~mm}$ for the DN group. Independent sample $t$ test postulated an insignificant statistical difference between the study groups ( $\mathrm{p}=0.3939)$. similarly, the mean apical deviation in SG was $1.126 \pm 0.41 \mathrm{~mm}$, compared to a mean of $1.22 \pm 0.459 \mathrm{~mm}$ in the $\mathrm{DN}$ group. Independent sample $\mathrm{t}$ test revealed statistically insignificant difference between the comparative groups.

On the other hand, the mean angular deviation in the SG group $3.13 \pm 1.28$ degree compared to $4.22 \pm 1.39$ degrees in the DN group. Independent sample $t$ test presented a statistically significant difference between the study groups (Mean difference $=-1.1 \mathrm{SE}=0.488, \mathrm{P}=0.032$, CI from -2.1026 to -0.0974$)$

TABLE (I): Coronal, Apical \& Angular deviation of both groups.

\begin{tabular}{|l|c|c|c|c|c|c|c|c|c|}
\hline & \multirow{2}{*}{ N } & \multicolumn{2}{|c|}{ SG } & \multicolumn{2}{c|}{ DN } & \multicolumn{1}{c|}{$\begin{array}{c}\text { Mean } \\
\text { difference }\end{array}$} & SE & P value & $\begin{array}{c}\text { Confidence } \\
\text { interval }\end{array}$ \\
\cline { 2 - 10 } & & Mean & SD & Mean & SD & & & \\
\hline Coronal deviation & 14 & 0.6307 & 0.2641 & 0.72 & 0.2805 & -0.0893 & 0.103 & 0.3938 & -0.3009 to 0.1224 \\
\hline Apical deviation & 14 & 1.1264 & 0.4113 & 1.2207 & 0.459 & -0.0943 & 0.165 & 0.572 & -0.4329 to 0.2443 \\
\hline Angular deviation & 14 & 3.1286 & 1.2887 & 4.2286 & 1.392 & -1.1 & 0.488 & 0.0328 & -2.1026 to -0.0974 \\
\hline
\end{tabular}






Fig. (8): Coronal \& Apical Linear deviation chart in both groups.

\section{DISCUSSION}

In the current research work, entire aspects that might disturb the osseointegration of implants were cautiously contemplated during patient selection. Those factors might be biological, mechanical or both. ${ }^{[35-37]}$

Fourteen patients of age extending from 35-45 years old were recruited with bounded partially edentulous spans in the maxilla in the current investigation to escape any variation in bone changes that might disturb the acquired outcomes. ${ }^{[38]}$

Any Uncooperative patients were omitted from the research, to ensure strict obligation to the oral hygiene measures and the consistent follow up schedules. ${ }^{[39]}$

Sufficient bone dimensions were assessed radiographically to warrant primary stability of the implant at the time of its placement. ${ }^{[40-42]}$

3D imaginings play a decisive role in implant navigation systems ${ }^{[43]}$. Studies revealed the efficacy of cone beam CT while planning of several dental approaches including implant positioning ${ }^{[22]}$. But the use of cone beam CT for assessment of dental implants revealed wide diversity of thoughts.

The expression "learning curve" denotes the buildup of skills through the duplication of any



Fig. (9): Angular deviation chart in both groups.

activity. However, no current study accurately postulated the effect of learning curves regarding implant navigation systems. ${ }^{[44]}$

In current study, we have tried to validate the accuracy and dependability of the implant navigation system to overcome limitations or shortcomings of the stable guide system regarding the heat generation during the drilling procedures, inaccessibility of drilling in posterior regions and finally, any error in planning procedures will be reflected on the actual implant positioning. ${ }^{[45-47]}$

The outcomes of the existent clinical research verified the null hypothesis previously created.

\section{CONCLUSION}

Regarding the limitations of the current study, involving the relatively small sample size, it might be stated that: Navigation Implantology system can afford high levels of accuracy with diminished noticeable error regarding implant positioning.

\section{CONFLICT OF INTEREST}

The current study exhibited Authors self-funding, without any conflict of interest. 


\section{REFERENCES}

1. Avrampou M, Mericske-Stern R, Blatz M, et al.: Virtual implant planning in the edentulous maxilla: criteria for decision making of prosthesis design. Clin Oral Impl Res 2013; 24 (Suppl. A100):152-159

2. Sanz M, Ivanoff CJ, Weingart D, et al: Clinical and radiologic outcomes after submerged and transmucosal implant placement with two-piece implants in the anterior maxilla and mandible: 3-year results of a randomized controlled clinical trial. Clin Implant Dent Relat Res 2015; 17:234-246

3. Andreiotelli M., Att W., Strub JR.: Prosthodontic complications with implant overdentures; a systematic literature review. Int. J. Prosthodont., 2010; 23:195-203.

4. Stoumpis C., Kohal RJ.: To splint or not to splint oral implants in the implant-supported overdenture therapy? A systematic literature reviews. J. Oral Rehabil. 2011; 38:857-869.

5. Papaspyridakos P., Chen CJ., Singh M., Weber HP., Gallucci GO.: Success criteria in implant dentistry; a systematic review. J. Dent. Res. 2012; 91:242-248.

6. Kohen J, Matalon S, Block J, et al.: Effect of implant insertion and loading protocol on long-term stability and crestal bone loss: a comparative study. J Prosthet Dent 2016; 115:697-702

7. Clelland N, Chaudhry J, Rashid RG, McGlumphy E.: Splitmouth comparison of splinted and non-splinted prostheses on short implants: 3-year results. Int. J Oral Maxillofac Implants. 2016;31(5):1135-1141.

8. Vervaeke S, Collaert B, De Bruyn H.: Immediate loading of implants in the maxilla: survival and bone loss after at least 2 years in function. Int J Oral Maxillofac Implants. 2013;28(1):216-221.

9. Klemetti E.: Is there a certain number of implants needed to retain an overdenture? J. Oral. Rehab. 2008; 35 (Suppl 1):80-84.

10. Al-Zubeidi MI., Alsabeeha NH., Thomson WM., Payne AG.: Patient satisfaction with maxillary 3-implant overdentures using different attachment systems opposing mandibular 2-implant overdentures. Clin. Implant Dent. Rel. Res. 2012; 14 (Suppl 1): e11-e19.

11. Guljé FL, Raghoebar GM, Vissink A, Meijer HJ.: Single crowns in the resorbed posterior maxilla supported by either 6-mm implants or by $11-\mathrm{mm}$ implants combined with sinus floor elevation surgery: a 1-year randomized controlled trial. Eur J Oral Implantol. 2014;7(3):247-255.

12. Guljé F, Abrahamsson I, Chen S, Stanford C, Zadeh H, Palmer R.: Implants of $6 \mathrm{~mm}$ vs. $11 \mathrm{~mm}$ lengths in the posterior maxilla and mandible: a 1-year multicenter randomized controlled trial. Clin Oral Implants Res. 2013;24(12):1325-1331.

13. Al Amri MD, Abduljabbar TS, Al-Kheraif AA, et al.: Comparison of clinical and radiographic status around dental implants placed in patients with and without prediabetes: 1-year follow-up outcomes. Clin Oral Implants Res 2017; 28:231-235

14. Brennan M., Houston F., O’Sullivan M., O'Connell B.: Patient satisfaction and oral health-related quality of life outcomes of implant overdentures and fixed complete dentures. Int. J. Oral Maxillofac Impl. 2010; 25:791-800.

15. Al Amri MD, Abduljabbar TS: Comparison of clinical and radiographic status of platform-switched implants placed in patients with and without type 2 diabetes mellitus: a 24-month follow-up longitudinal study. Clin Oral Implants Res2017; 28:226-230

16. Fromentin O., Lassauzay C., Abi Nader S., Feine J., de Albuquerque Junior RF.: Testing the retention of attachments for implant overdentures - validation of an original force measurement system. J. Oral Rehab. 2010; 37:54-62.

17. Al Amri MD: Crestal bone loss around submerged and non-submerged dental implants: a systematic review. J Prosthet Dent 2016; 115:564-570.e1

18. Calvo-Guirado JL, Perez-Albacete C, Aguilar-Salvatierra A, et al.: Narrow- versus mini-implants at crestal and subcrestal bone levels. Experimental study in beagle dogs at three months. Clin Oral Investig 2015; 19:1363-1369

19. Hasan, I, Dominiak M, Blaszczyszyn A, Bourauel C, Gedrange T, Heinemann F.: Radiographic evaluation of bone density around immediately loaded implants. Ann Anat. 2015; 199:52-57.

20. Tatli U, Salimov F, Kürkcü M, Akoğlan M, Kurtoğlu C. Does cone beam computed tomography-derived bone density give predictable data about stability changes of immediately loaded implants? a 1-year resonance frequency follow-up study. J Craniofac. Surg. 2014; 25:293-299. 
21. Aziz AS, Kalekar MG, Suryakar AN, et al: Assessment of some biochemical oxidative stress markers in male smokers with chronic periodontitis. Indian J Clin Biochem. 2013 ; 28:374-380

22. Bidra AS: Three-dimensional esthetic analysis in treatment planning for implant-supported fixed prosthesis in the edentulous maxilla: review of the esthetics literature. J. Esthet. Rest. Dent. 2011; 23:219-236.

23. Rohlin M., Nilner K., Davidson T., et al.: Treatment of adult patients with edentulous arches: a systematic review. Int. J. Prosthod. 2012;.25:553-567.

24. Sadowsky SJ., Bedrossian E.: Evidenced-based criteria for differential treatment planning of implant restorations for the partially edentulous patient. J. Prosthod. 2013; 22:319-329.

25. Al Amri MD, Kellesarian SV, Al-Kheraif AA, et al: Effect of oral hygiene maintenance on HbAlc levels and periimplant parameters around immediately-loaded dental implants placed in type- 2 diabetic patients: 2 years followup. Clin Oral Implants Res 2016; 27:1439-1443

26. Fortin T., Camby E., Alik M., Isidori M., et al: Panoramic images versus three-dimensional planning software for oral implant planning in atrophied posterior maxillary; a clinical radiological study. Clin. Impl. Dent. Rel. Res. 2013; 15:198-204.

27. Esposito M, Grusovin MG, Maghaireh H, Worthington HV:: Interventions for replacing missing teeth: different times for loading dental implants. Cochrane Database Syst. Rev. 2013;3:CD003878.

28. Kutan E, Bolukbasi N, Yildirim-Ondur E, et al.: Clinical and radiographic evaluation of marginal bone changes around platform-switching implants placed in crestal or sub-crestal positions: a randomized controlled clinical trial. Clin Implant Dent Relat. Res 2015;17(Suppl 2): e364-375

29. Katsoulis J., Brunner A., Mericske-Stern R.: Maintenance of implant-supported maxillary prostheses: a 2-year controlled clinical trial. Int. J. Oral Maxillofac. Impl. 2011; 26:648-656.

30. Aimetti M, Ferrarotti F, Mariani GM, et al.: Soft tissue and crestal bone changes around implants with platformswitched abutments placed non-submerged at sub-crestal position: a 2-year clinical and radiographic evaluation. Int. J Oral Maxillofac Implants 2015; 30:1369-1377
31. Chrcanovic BR, Albrektsson T, Wennerberg A: Immediately loaded non-submerged versus delayed loaded submerged dental implants: a meta-analysis. Int. J Oral Maxillofac Surg. 2015; 44:493-506

32. Becker W.: Osseointegration: have we tinkered with the process too much? Clin. Impl. Dent. Rel. Res. 2012; 14 (6):779-780.

33. Kheur MG, Sandhu R, Kheur S, Le B, Lakha T.: Reliability of resonance frequency analysis as an indicator of implant micromotion: an in vitro study. Implant Dent. 2016; 25:783-788.

34. Huang H, Wismeijer D, Shao X, Wu G.: Mathematical evaluation of the influence of multiple factors on implant stability quotient values in clinical practice: a retrospective study. Ther Clin Risk Manag. 2016; 12:1525-1532.

35. Kim SJ, Ribeiro AL, Atlas AM, et al.: Resonance frequency analysis as a predictor of early implant failure in the partially edentulous posterior maxilla following immediate nonfunctional loading or delayed loading with single unit restorations. Clin Oral Implants Res. 2015; 26:183-190.

36. Slot W., Raghoebar GM., Vissink A., et al.: Maxillary overdentures supported by four or six implants in the anterior region; 1-year results from a randomized controlled trial. J. Clin. Period. 2013; 40:303-310.

37. Slot W., Raghoebar GM., Vissink A., et al.: A systematic review of implant-supported maxillary overdentures after a mean observation period of at least 1 year. J Clin. Period. 2010; 37:98-110.

38. Grassi R., Rebaudi A., Trisi P., Covani U., Barone A.: Bone loss around immediately loaded transitional implants: histologic and micro-computed tomographic analysis--a case report. Int. J. Period. Rest. Dent. 2012; 32 (6):195-203.

39. Trisi P, Berardini M, Falco A, Podaliri Vulpiani M.: Validation of value of actual micromotion as a direct measure of implant micromobility after healing (secondary implant stability). An in vivo histologic and biomechanical study. Clin Oral Implants Res. 2016; 27:1423-1430.

40. Osman RB., Payne AG., Ma S .: Prosthodontic maintenance of maxillary implant overdentures: a systematic literature review. Int. J. Prosth. 2012; 25:381-391.

41. Gobbato L., Avila-Ortiz G., Sohrabi K., et al.: The effect of keratinized mucosa width on peri-implant health: A systematic review. Int. J. Oral Maxillofac. Impl. 2013; 28:1536-1545. 
42. Cesaretti G, Botticelli D, Renzi A, Rossi M, Rossi R, Lang NP.: Radiographic evaluation of immediately loaded implants supporting 2-3 units fixed bridges in the posterior maxilla: a 3-year follow-up prospective randomized controlled multicenter clinical study. Clin Oral Implants Res. 2016; 27:399-405.

43. Böhringer, S. (2016). Accuracy investigation of a new mininavigation system for dental implants. Biomed Tech 61(s1), S115.

44. D'Haese, J. (2012). Accuracy and complications using computer-designed stereo-lithographic surgical guides for oral rehabilitation by means of dental implants: a review of the literature. Clin Implant Dent Relat Res 14(3), 321-33.

45. Gulati, M. (2015). Computerized implant-dentistry: Advances toward automation. J Indi-an Soc Periodontol, 5-10.

46. Hemm, S. (2014). Diamond-like carbon coating for highly precise and biocompatible optical markers for surgical navigation. Biomedizinische Technik, 48th DGBMT Annual Conference, (S. DOI: 10.1515/bmt-2014-4187).

47. Wittneben, J.-G. (2014). Complication and Failure Rates with Implant-Supported Fixed Dental Prostheses and Single Crowns: A 10-Year Retrospective Study. Clin Implant Dent Res, 356-364. 